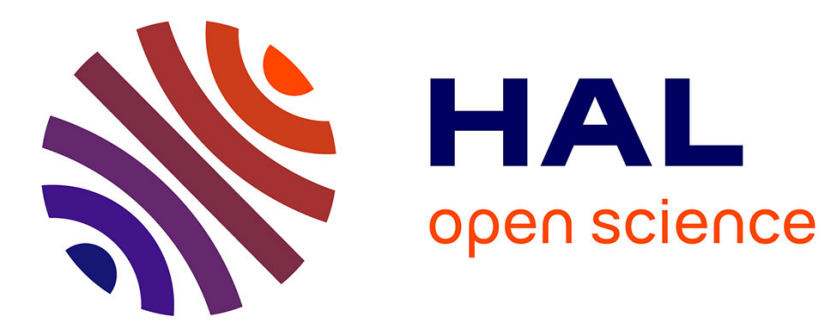

\title{
Evaluation and design: a knowledge-based approach
}

\author{
Élise Vareilles, Michel Aldanondo, Paul Gaborit
}

\section{To cite this version:}

Élise Vareilles, Michel Aldanondo, Paul Gaborit. Evaluation and design: a knowledge-based approach. International Journal of Computer Integrated Manufacturing, 2007, 20 (7), p. 639-653. 10.1080/09511920701566517. hal-01599444

\section{HAL Id: hal-01599444 \\ https://hal.science/hal-01599444}

Submitted on 17 Oct 2017

HAL is a multi-disciplinary open access archive for the deposit and dissemination of scientific research documents, whether they are published or not. The documents may come from teaching and research institutions in France or abroad, or from public or private research centers.
L'archive ouverte pluridisciplinaire HAL, est destinée au dépôt et à la diffusion de documents scientifiques de niveau recherche, publiés ou non, émanant des établissements d'enseignement et de recherche français ou étrangers, des laboratoires publics ou privés. 


\title{
Evaluation and design: a knowledge-based approach
}

\author{
É. VAREILLES*, M. ALDANONDO and P. GABORIT \\ Centre de Génie Industriel, École des mines d'Albi-Carmaux, Campus Jarlard, \\ 81013 Albi CT Cedex 09, France
}

\begin{abstract}
The aim of this communication is to describe how aiding-design tools can evaluate designed solutions to help users make the best choices, avoid design mistakes and reduce the design time-cycle. First, we will compare the two main methods for aiding designbehaviour simulation tools and domain knowledge simulation tools - and look at their advantages and drawbacks. We will focus on tools based on knowledge because of their 'interactivity' and for their ability to represent domain knowledge and show how they can be extended to evaluate designed solutions. We will then concentrate on an aiding-design tool based on constraints and see how a solution can be evaluated using an evaluation function. As such a tool has already been developed as part of a European project to help metallurgists design and evaluate heat treatment operations, we end with the presentation of a real example.
\end{abstract}

Keywords: Evaluation of solutions; Interactive aiding design; Constraints; Application

\section{Introduction}

It is very important in aiding design to know the relevance of the solutions obtained. Indeed, it is necessary to know if one solution is better than another in order to make a good decision. An estimation of the relevance of the solutions is therefore essential. We mean by estimating a solution an evaluation of its quality, its performance and its efficiency. The question of the relevance of the solutions is even more crucial in the context of integrated design where designers come from different domains and have different (and, most of the time, conflicting) objectives (Huang 1996, Magrab 1997), e.g. the maximization of the performance and quality of a product and, simultaneously, the minimization of costs. The solution must, at best, respect the different objectives.

There are two main ways of helping users make choices by giving an idea of the behaviour of systems and characterizing the relevance of solutions: behaviour simulation, which is mainly based on mathematical expressions, and domain knowledge simulation, which is mainly based on 'know-how' and experimental knowledge. These two approaches can evaluate the relevance of a solution as follows.

- Behaviour simulations, for instance event-driven simulations (Banks et al. 1984), finite element models (Szabo and Babuska 1991), or models based on virtual reality, consider the evolution of a problem through time. They work well when problems have a temporal or physical behaviour that can be modelled by mathematical formulae. In general, the solutions returned by behaviour simulations are optimized following several criteria $(\mathrm{Fu} 2002)$ which lead to one of the optimal solutions; the relevance of the solutions is quantitative. The process of behaviour simulation can be divided into three steps. First, the user has to design his/her solution to the problem; secondly, (s)he has to simulate its behaviour; and, finally, (s)he has to decide if the solution is the relevant one or not. If not, (s)he has to re-design the problem and loops, as shown in the left part of figure 1. The aiding-design process is then a trial and error one.

*Corresponding author. Email: vareille@enstimac.fr 


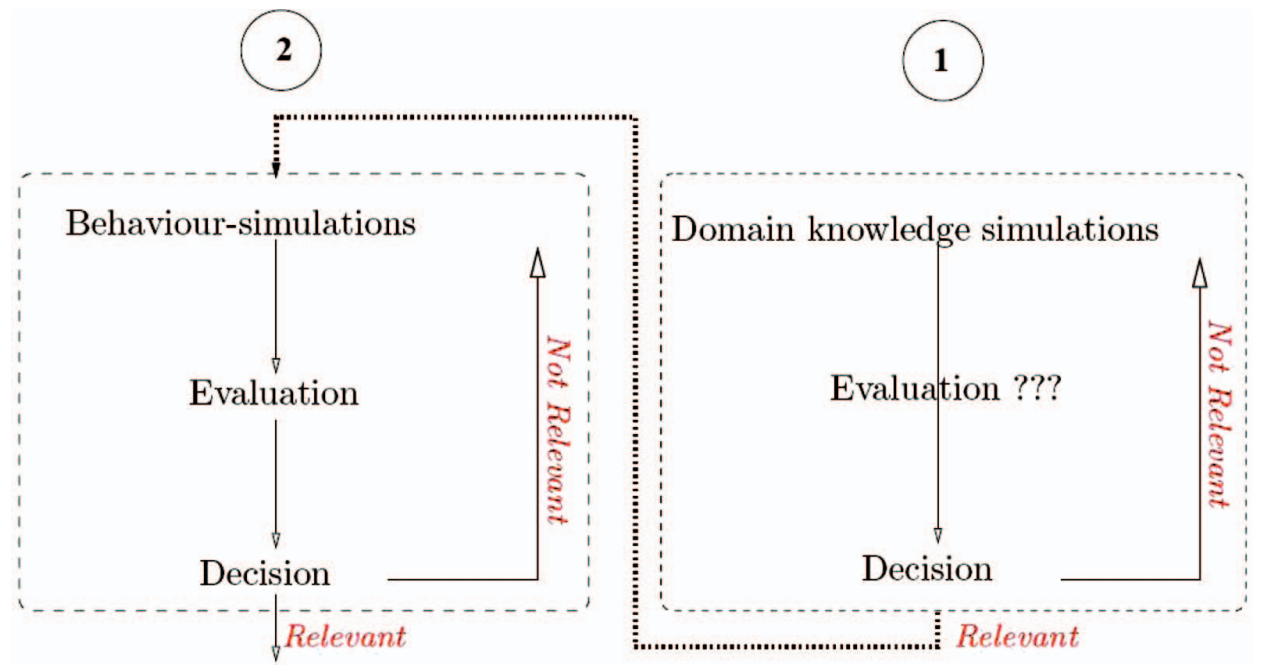

Figure 1. Knowledge and simulation aiding-design tools.

- Domain knowledge simulations, for instance casebased reasoning (Riesback and Shank 1989), expert systems (Buchanan and Shortliffe 1984) and constraint satisfaction problems (Montanari 1974), are mainly based on the 'know-how' of experts and provide experts' advice. Experts' know-how is domain knowledge, resulting from the experts' skills and abilities. This kind of knowledge cannot be extracted and modelled easily. Usually, the solutions returned by domain knowledge simulation give approximate ideas of the result; the relevance of the solutions is qualitative. The process of using domain knowledge simulation can be divided into two steps: firstly, the user is guided step by step by the experts' advice in order to design his/her problem and finally an approximate idea of the solution is found. Then the user has to decide if the solution is the relevant one or not. If not, (s)he has to re-design the problem by making different choices respecting the experts' advice, as shown in the right side of figure 1 . The aiding-design process is more an interactive one.

Behaviour simulations are mainly used to optimize solutions, whereas domain knowledge simulations are mainly used to assist decisions in an interactive way. These two methods are not in conflict with each other and can be used to complement one another in different ways (Tsatsoulis 1990). For instance, and as shown in figure 1, the user can start with a domain knowledge simulation in order to design and estimate the solutions of her/his problem and then (s)he can use a behaviour simulation to obtain a more accurate result.
The aim of this paper is to show how the relevance of the solutions can be estimated or evaluated in domain knowledge simulations in order to help users make better decisions. We concentrate on the tools based on knowledge because of their 'interactivity' in aiding the design process and their ability to represent domain knowledge, and we study how they can be extended to evaluate the designed solutions. In section 2 we will look at different domain knowledge simulation tools and different methods of evaluating solutions. In section 3 we will concentrate on aiding-design tools based on constraints because of their consistent properties and see how a solution can be evaluated in such a case. Section 4 will illustrate our proposition with a real example.

\section{Knowledge-based systems and evaluation functions}

There are several ways of modelling an expert's knowledge to use it in aiding the design process. Experts' know-how can either be implicitly expressed (embodied in past cases, for instance) or explicitly expressed (as mathematical expressions). Several methods of evaluating the relevance of solutions also exist and can be determined directly by experts or learned from past experiments. If the relevance of the solutions corresponds to a set of simple and independent data, it can be directly embodied in the system; if not, a method of evaluating the relevance of the solution must be added.

\subsection{Implicit knowledge}

In case-based reasoning (CBR) (Maher et al. 1995), expertise is embodied in a library of past cases, rather than 
being encoded in classical rules. Each case typically contains a description of the problem, plus a solution and/or the outcome. The knowledge and reasoning process used by experts to solve the problem is not recorded, but is implicit in the solution. In order to find a solution, the user describes her/his problem through a list of parameters and, after all the user's inputs, the problem described is matched against the cases in the data base. A similarity function (Kolodner 1993) makes it possible to detect and classify similar cases and the most similar or the most adaptable ones are retrieved. We should point out that if the user's problem does not match any past case, the system will return the nearest possible cases. The cases retrieved provide ballpark solutions that must generally be adapted by the user to fit her/his current problem.

\subsection{Explicit Knowledge}

In expert systems (ES) (Buchanan and Shortliffe 1984), experts' knowledge is explicitly expressed as 'inference rules'. An inference rule is a statement that has two parts: an if-clause and a then-clause. For instance, let us consider the disjunctive syllogism: if $[p \vee k]$ then $[q]$. An expert system is made up of many such inference rules. An inference engine uses the inference rules to draw conclusions. There are two main methods of reasoning when using inference rules: forward and backward chaining. Forward chaining starts with any available data in the if-clause and uses the inference to conclude more data, until the desired goal corresponding to a then-clause is reached. Backward chaining starts with a list of goals corresponding to a thenclause and works backwards to see if there are data in the if-clause which allow it to conclude any of these goals. The two methods of reasoning can be used simultaneously.

In constraint satisfaction problems (CSP) (Tsang 1993), experts' knowledge is explicitly expressed as constraints, such as lists of permissible combinations, $\{(x=1, y=a)$, $(x=2, y=b), \ldots\}$, mathematical formulae, $y=x^{4}+3 \times x$, or logical rules, $(A \vee B) \wedge C$. All the constraints are gathered in a model which corresponds to the experts' knowledge concerning the domain. A model contains a set of variables denoted $X$, with definition domains denoted $D$, and a set of compatibility constraints denoted $C$, expressing the permissible combinations of values of the variables. In order to find a solution, the user describes her/his problem through the model variables. The method consists of reflecting the user's inputs through the constraints network to the other variables by limiting their domains to consistent values. This mechanism, repeated several times, restricts the solution space progressively to reach coherent solutions.

The most difficult points in expert systems and in constraint satisfaction problems are the extraction of the experts' know-how, its translation into, respectively, inference rules and constraints, and its validation.

\subsection{Evaluation of solutions}

We identify two different ways of dealing with the evaluation of the relevance of solutions in domain knowledge simulation tools:

- the evaluation of a solution can be embodied in the aiding-design system if it results from a set of simple and independent data;

- the method of evaluating a solution can be added to the aiding-design model if the relevance of the solutions is due to a huge set of correlated data. In this case, we say that the evaluating method is added to the system.

2.3.1. Evaluation as part of the system. In previous approaches the evaluation of the solutions could be embodied in the system if it corresponded to a set of simple and independent data, such as a set of physical measures.

In CBR, the evaluation could be a part of the description of past cases by adding particular parameters corresponding to the set of data the user needs to focus on in order to evaluate the solutions. In this case, all the most similar past cases are retrieved with their evaluations.

In ES and CSP, the evaluation could be a set of particular rules or constraints that determines if a set of conclusions or variables is evaluated to specific values.

If a huge set of correlated data has to be taken into account in order to evaluate the solutions, an analytical study must first be performed by an expert to classify the solutions stored in previous approaches. If this work is not done, only an expert user will be able to compare the different solutions.

2.3.2. Evaluation as an addition to the system. A means of evaluation can be added to the design models in order to evaluate solutions. This can be established by the 'knowhow' of experts and deduced from past experiments.

If the experts can determine the set of data to take into account and how to evaluate a solution from them by their aggregation, an evaluation function can be expressed as a rough mathematical formula to compute a qualitative evaluation. The evaluation function is then added to the system.

If an evaluation function cannot be expressed as a mathematical formula or cannot be defined by the experts, the method of evaluating solutions can be learned from past experiments and from experts' knowledge by an artificial neural network (ANN) or by a regression analysis. An ANN 
is composed of a large number of interconnected processing layers of neurons working in unison to solve a specific problem through a learning process. The basic element of an ANN is the perceptron (Minsky and Papert 1969): the perceptron itself has five basic elements: an $n$-vector input, weights assigned to each neuron connection, a summing function, a threshold device and an output. Knowledge is learned from past cases and weights are adjusted so that, given a set of inputs and experts' knowledge, the associated connections will produce the desired output, in this case a qualitative evaluation of the solution.

Regression analysis is a statistical tool for the investigation of the relationship between variables. Usually, the investigator seeks to ascertain the causal effect of one variable upon another. To explore such issues, the investigator assembles data on the underlying variables of interest and employs a regression to estimate the quantitative effect of the causal variables upon the variable that they influence. The investigator also assesses the 'statistical significance' of the estimated relationship, i.e. the degree of confidence that the true relationship is close to the estimated one.

\subsection{Synthesis}

Experts' knowledge can either be implicitly expressed, as in CBR, or explicitly expressed, as in ES and CSP, and used in aiding the design process. There are several methods for evaluating the relevance of solutions that can be determined directly by experts or learned from past experiments.

If the evaluation of the relevance of solutions results from a small set of relevant and independent data, it can be directly embodied in the system as a particular parameter in CBR, a particular rule in ES and a particular variable in CSP. If it results from a huge set of correlated data, an evaluation method must be added to the system, such as an explicit evaluation function defined by experts, an ANN or regression analysis. All these methods can be connected to previous knowledge-based systems.

\section{Aiding design and evaluation with constraints}

We focus on aiding-design models based on constraints (CSP) because, unlike CBR and ES, they, firstly, allow a diversity of representations of domain knowledge, and, secondly, they can guarantee some consistency in users' inputs during the search for a solution (Dechter 1992). As this kind of tool is based on the extraction of knowledge, we assume that the evaluation function can be extracted and formalized as a mathematical formula during the extraction of the experts' know-how.

An aiding-design tool based on constraints corresponds to a design model and a model of the evaluation function, as shown in figure 2. We assume that these two parts can be explicitly formulated from experts' know-how. Therefore, two types of variables are considered (Vernat 2004): those belonging to the design model, denoted $\left\{V_{\mathrm{d}}\right\}$, and those belonging to the evaluation function, denoted $\left\{V_{\mathrm{e}}\right\}$, as shown in figure 2 . The design variables $\left\{V_{\mathrm{d}}\right\}$ correspond to the experts' domain knowledge and also to technical feasibility, whereas the evaluation variables $\left\{V_{\mathrm{e}}\right\}$ are used to compute the evaluation mark $v_{\mathrm{ef}}$ of the solutions.

\subsection{Architecture of a constraints-based model}

The design variables $\left\{V_{\mathrm{d}}\right\}$ can be either discrete or continuous depending on the cardinal attribute of their domains. The domains of discrete variables are countable and defined as lists of symbols, integers or floats or as intervals of integers, whereas the domains of continuous variables are uncountable and defined as intervals of floats (Vareilles 2005). For instance, let $v_{1}$ be a numerical discrete variable with domain $\{1,2,[5,45], 70\}$.

The design variables are linked with constraints such as mathematical formulae or compatibility tables which list all the permissible combinations of values for a set of variables. Let us look at the first compatibility table, $c_{1}$, of table 1. This compatibility table involves a pair of variables $v_{\mathrm{d} 2}$ and $v_{\mathrm{d} 3}$ and tells us that the only two permissible combinations of values for this pair are $(\perp,<20)$ and $(\|, \geq 20)$. These compatibility tables can be either discrete if they link only discrete variables, continuous if they link only continuous variables or mixed if they link discrete and continuous variables, as in our previous example (Gelle 1998).

The evaluation variables $\left\{V_{\mathrm{e}}\right\}$ are continuous and defined within intervals of floats. The evaluation variables $\left\{V_{\mathrm{e}}\right\}$ are linked by the evaluation function that computes the evaluation mark of a solution, the continuous variable $v_{\text {ef. }}$.

The design $\left\{V_{\mathrm{d}}\right\}$ and the evaluation $\left\{V_{\mathrm{e}}\right\}$ variables are most often linked together with mixed compatibility tables. Indeed, the combinations of values of the design variables has an impact on the evaluation mark of a solution.

Let us consider an example from the industrial problem that is at the heart of our work: an axis with an inner hole, as shown in figure 3, the skin hardness of which must be improved by quenching in a particular medium. Unfortunately, during quenching, a negative effect corresponding to part distortion generally occurs. This occurrence depends on the design of the quenching operation. In this example we focus on the effect (1) of gravity, (2) of the quenching fluid direction - it can be either parallel or perpendicular to the part axis - and (3) of the position of the inner hole on the distortion intensity, which, in this particular problem, is the relevant data chosen to evaluate the quenching operation. 


\section{Design model}

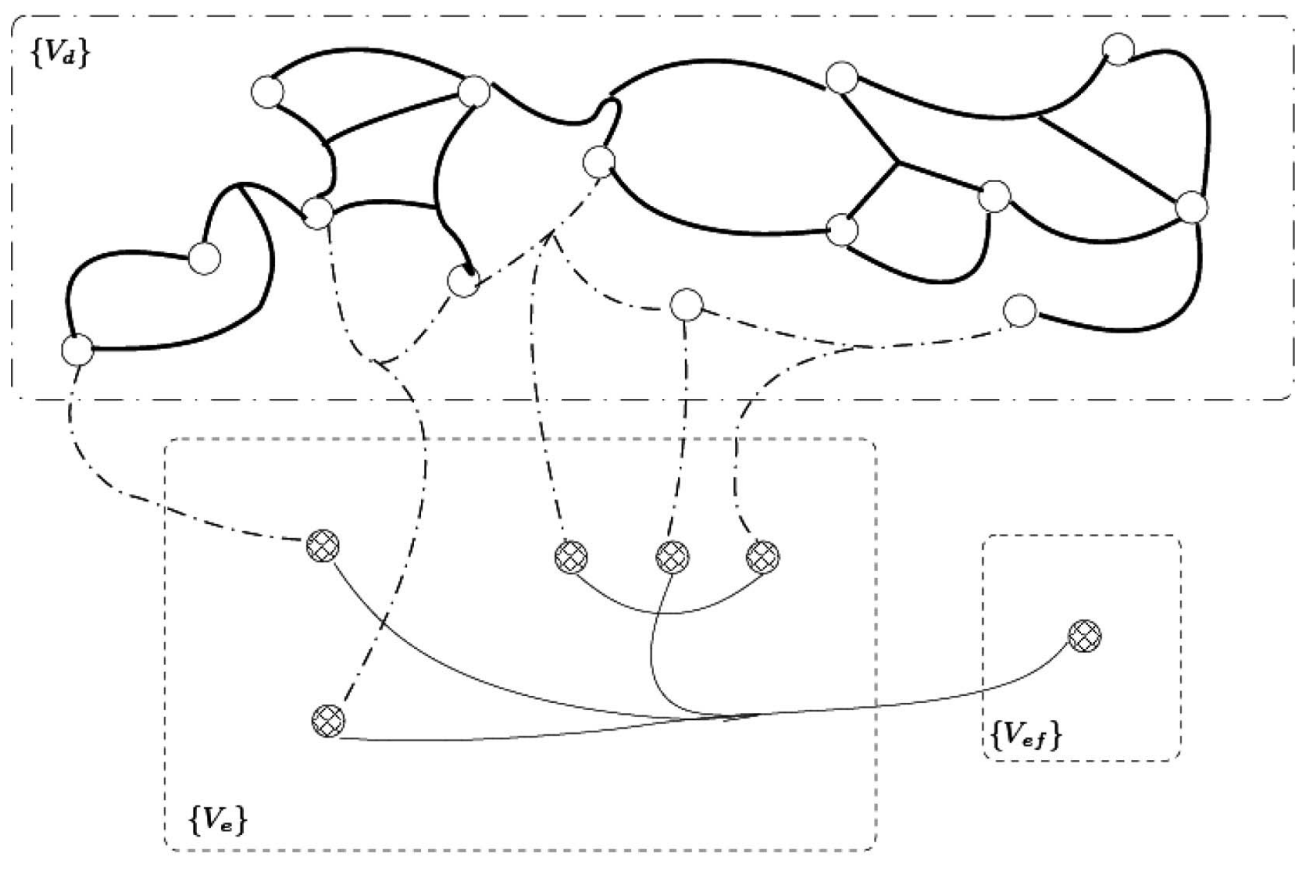

Evaluation function

Caption:

Design variables

Evaluation variables

Relations linking the design variables

-.- Relations linking the design variables

to the evaluation variables

— Relations linking the evaluation variables

Figure 2. Global architecture.

Table 1. Compatibility constraint of the real example.

\begin{tabular}{|c|c|c|c|c|c|c|}
\hline \multicolumn{2}{|c|}{$c_{1}$} & \multicolumn{3}{|c|}{$c_{2}$} & \multicolumn{2}{|c|}{$c_{3}$} \\
\hline$v_{\mathrm{d} 2}$ & $v_{\mathrm{d} 3}$ & $v_{\mathrm{d} 1}$ & $v_{\mathrm{d} 2}$ & $v_{\mathrm{e} 1}$ & $v_{\mathrm{d} 3}$ & $v_{\mathrm{e} 2}$ \\
\hline$\perp$ & $<20$ & $\perp$ & $\perp$ & {$[7,10]$} & $<20$ & {$[1,2]$} \\
\hline$\|$ & $\geq 20$ & $\perp$ & $\|$ & {$[4,7]$} & $\geq 20$ & {$[2,3]$} \\
\hline & & $\|$ & $\perp$ & {$[4,7]$} & & \\
\hline & & $\|$ & $\|$ & {$[1,4]$} & & \\
\hline
\end{tabular}

The associated network, presented in figure 4, is composed of:

- three design variables belonging to $\left\{V_{\mathrm{d}}\right\}$ : two symbolic variables, $v_{\mathrm{d} 1}$ corresponding to the direction of gravity versus the part axis, with domain $D_{v_{\mathrm{d} 1}}=$ $\{\perp, \|\}$, and $v_{\mathrm{d} 2}$, corresponding to the direction of the quenching fluid versus the axis, with domain $D_{v_{\mathrm{d} 2}}=\{\perp, \|\}$, and one numerical discrete variable $v_{\mathrm{d} 3}$ corresponding to the axis gap, with domain $D_{v_{\mathrm{d} 3}}=\{[0,+\infty\}$;

- three evaluation variables $v_{\mathrm{e} 1}, v_{\mathrm{e} 2}$ and $v_{\mathrm{ef}}$ with domains $\quad D_{v_{\mathrm{e} 1}}=\{[1,10]\}, D_{v_{\mathrm{e} 2}}=\{[1,3]\}$ and $D_{v_{\mathrm{ef}}}=$ $\{[1,30]\}$, respectively, which allow computation of the intensity of distortion;

- three compatibility tables, presented in table 1 :

- one, $c_{1}$, which is a mixed constraint between two design variables $v_{\mathrm{d} 2}$ and $v_{\mathrm{d} 3}$ corresponding to the fact that the axis gap should be less than 20 if the quenching direction is perpendicular to the part axis, and greater than 20 otherwise, 


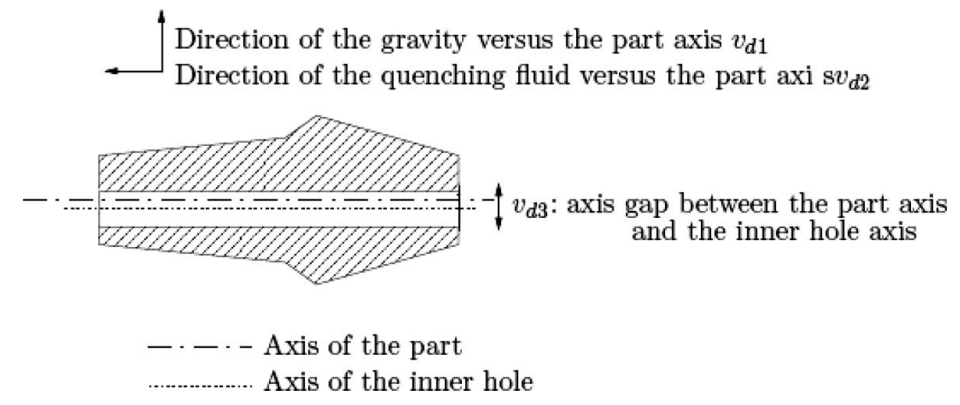

Figure 3. Shaft example.

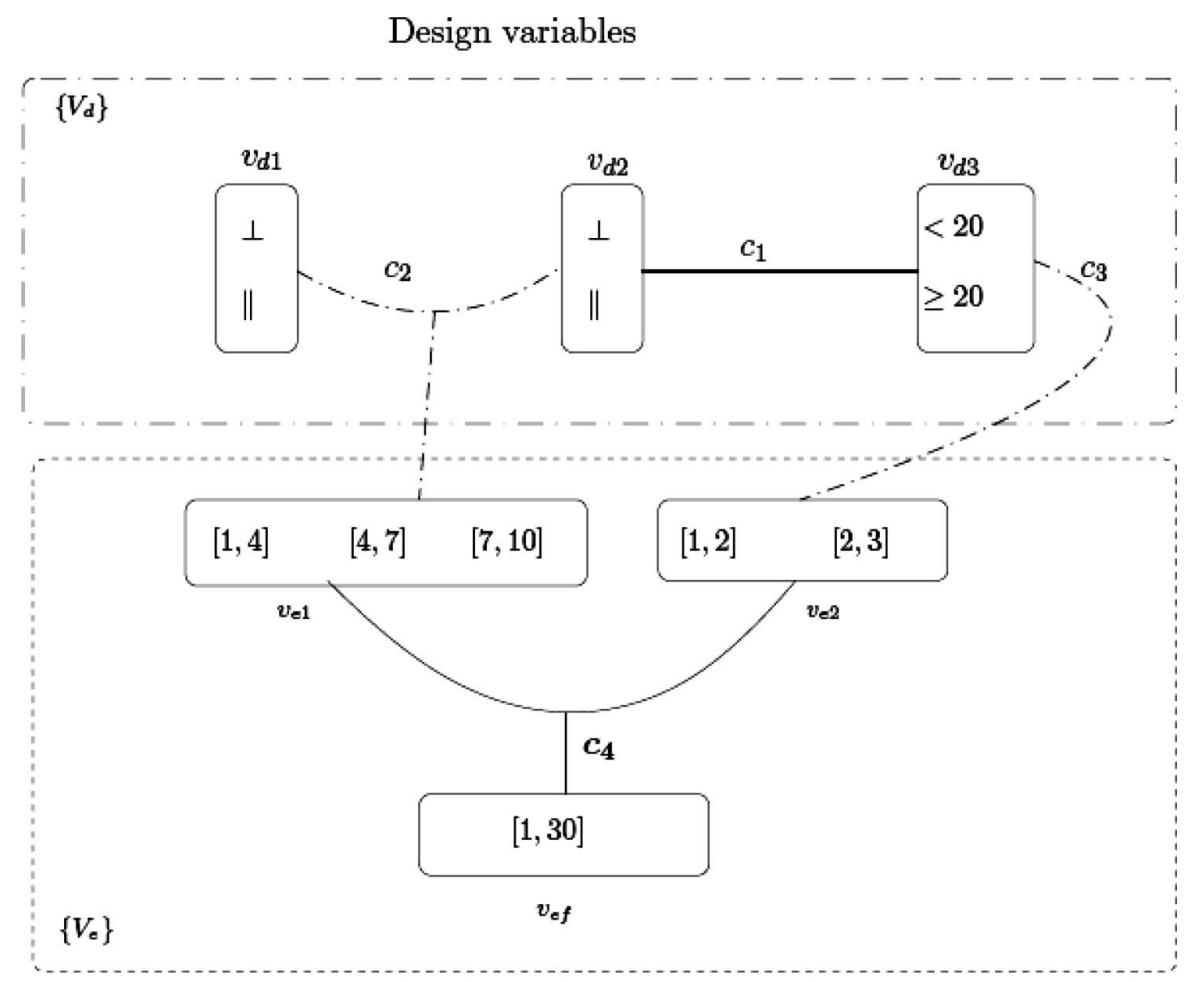

Variables of evaluation

Caption:

— Constraints linking the design variables

-.- Constraints linking the design variables

to the variables of evaluation

- Constraints linking the variables of evalutaion

Figure 4. Reasoning model example.

- one, $c_{2}$, which is a mixed constraint between two design variables $v_{\mathrm{d} 1}$ and $v_{\mathrm{d} 2}$ and an evaluation variable $v_{\mathrm{e} 1}$ stating that the worst case corresponds to the gravity and quenching fluid directions being perpendicular to the part axis, whereas the best case is where both are parallel to it,

- one, $c_{3}$, which is a mixed constraint between the design variable $v_{\mathrm{d} 3}$ and an evaluation variable $v_{\mathrm{e} 2}$, stating that the worst case corresponds to an axis 
gap greater than 20 , whereas the best case is where it is less than 20 ;

- one mathematical constraint, $c_{4}$, computing the evaluation mark:

$$
v_{\mathrm{ef}}=v_{\mathrm{e} 1} \times v_{\mathrm{e} 2} .
$$

\subsection{Operating modes}

Interactive aiding design consists of giving a value to or limiting the domain of a design variable $v_{\mathrm{d}}$. Modification of the domain of $v_{\mathrm{d}}$ is reflected through the constraints network to the other design variables by retrieving all the values from their domain that are now inconsistent with the reduced domain of $v_{\mathrm{d}}$. This mechanism, repeated several times, progressively restricts the solution space in order to reach a solution. In parallel, the evaluation mark is computed after each user's input. With aiding design being interactive, we use the filtering techniques of constraints programming:

- discrete, mixed and continuous compatibility tables are filtered using arc-consistency (Mackworth 1977, Faltings 1994);

- mathematical formulae are filtered using 2B-consistency (Lhomme 1993), based on interval arithmetic (Moore 1966). Interval arithmetic extends real arithmetic to intervals by applying the operators of a formula to the endpoints of the intervals of its arguments. For example, if we consider the constraint $f:(x, y) \mapsto x \times y$ that adds the variables $x$ to $D_{x}=[\underline{x}, \bar{x}]$ and $y$ to $D_{y}=[\underline{y}, \bar{y}]$, the result of $f$ yields $D_{x} \otimes D_{y}=$

$$
\begin{aligned}
& {[\min \{(\underline{x} \times \underline{y}),(\bar{x} \times \underline{y}),(\underline{x} \times \bar{y}),(\bar{x} \times \bar{y})\},} \\
& \max \{(\underline{x} \times \underline{y}),(\bar{x} \times \underline{y}),(\underline{x} \times \bar{y}),(\bar{x} \times \bar{y})\}] .
\end{aligned}
$$

Being based on constraints, which do not have propagating directions, the knowledge model can be used in two operating modes. The first, called evaluation of a solution, consists of interactively inputting some restrictions on the design variables $\left\{V_{\mathrm{d}}\right\}$ in order to find a consistent solution, simultaneously computing the evaluation mark $v_{\mathrm{ef}}$ of this solution and comparing it with another one. The second mode, called choices deduced from an evaluation, consists of inputting restrictions on non-negotiable design variables belonging to $\left\{V_{\mathrm{d}}\right\}$ and a threshold on the evaluation mark $v_{\mathrm{ef}}$ in order to deduce the value of the negotiable design variables of $\left\{V_{\mathrm{d}}\right\}$. These two operating modes are illustrated by the example presented in section 3.1 and by figure 3 .

3.2.1. Evaluation of a solution. In the first operating mode, the user restricts the domain of the design variables $\left\{V_{\mathrm{d}}\right\}$ step by step to find a solution, the evaluation mark $v_{\text {ef }}$ of which is computed simultaneously.
Let us consider the example presented in subsection 3.1. At the start, as no choice has been made, the evaluation mark $v_{\mathrm{ef}}$ equals $v_{\mathrm{ef}}=v_{\mathrm{e} 1} \times v_{\mathrm{e} 2}=[1,10] \times[1,3]=[1,30]$.

If the user reduces the design variable $v_{\mathrm{d} 1}$ to $\perp$, this reduction has a direct impact through the mixed constraint $c_{2}$ on the evaluation variable $v_{\mathrm{e} 1}$, which is reduced to $[4,10]$.

The reduction of the evaluation variable $v_{\mathrm{e} 1}$ to $[4,10]$ has an impact through the numerical constraint $c_{4}$ on the evaluation mark $v_{\mathrm{ef}}: v_{\mathrm{ef}}$ is reduced to $\left(v_{\mathrm{e} 1} \times v_{\mathrm{e} 2}\right) \cap D_{v_{\mathrm{ef}}}=$ $([4,10] \otimes[1,3]) \cap[1,30]=[4,30]$.

If the user reduces the design variable $v_{\mathrm{d} 2}$ to $\perp$, this reduction has a direct impact through the mixed constraint $c_{2}$ on the evaluation variable $v_{\mathrm{e} 1}$, which is reduced to $[7,10]$.

The reduction of the design variable $v_{\mathrm{d} 2}$ to $\perp$ has an impact through the constraint $c_{1}$ on the design variable $v_{\mathrm{d} 3}$, which is reduced to $<20$.

The reduction of the design variable $v_{\mathrm{d} 3}$ to $<20$ has an impact through the mixed constraint $c_{3}$ on the evaluation variable $v_{\mathrm{e} 2}$, which is reduced to $[1,2]$.

This reduction is reflected in the evaluation mark $v_{\mathrm{ef}}$ through the numerical constraint $c_{4}$ and reduces it to $\left(v_{\mathrm{e} 1} \times v_{\mathrm{e} 2}\right) \cap D_{v_{\mathrm{ef}}}=([7,10] \otimes[1,2]) \cap[4,30]=[7,20]$.

As the model is very simple, with only two user inputs, the problem is solved, $v_{\mathrm{d} 1}=\perp, v_{\mathrm{d} 2}=\perp, v_{\mathrm{d} 3}<20$, and its relevance is evaluated to $[7,20]$. This solution is rather bad because we want to minimize the intensity of distortion and, in the worst case $\left(v_{\mathrm{d} 1}=\perp, v_{\mathrm{d} 2}=\|, v_{\mathrm{d} 3} \geq 20\right)$ the relevance is evaluated to $[8,21]$ and in the best case $\left(v_{\mathrm{d} 1}=\|\right.$, $\left.v_{\mathrm{d} 2}=\|, v_{\mathrm{d} 3} \geq 20\right)$ the relevance is evaluated to $[2,12]$.

3.2.2. Choices deduced from an evaluation. In the second operating mode, the user first gives a value to the nonnegotiable design variables of $V_{\mathrm{d}}$ and then a threshold on the evaluation mark $v_{\mathrm{ef}}$ in order to deduce the value of the negotiable design variables belonging to $V_{\mathrm{d}}$.

For instance, let us assume that the part geometry cannot be changed, therefore the axis gap is a nonnegotiable variable. If the user reduces $v_{\mathrm{d} 3}$ to $<20$, this reduction has an impact through the constraint $c_{1}$ on the design variable $v_{\mathrm{d} 2}$, which is reduced to $\perp$, and through the constraint $c_{3}$ on the evaluation variable $v_{\mathrm{e} 2}$, which is reduced to $[1,2]$.

The reduction of $v_{\mathrm{d} 2}$ to $\perp$ is reflected through the constraint $c_{2}$ on the evaluation variable $v_{\mathrm{el}}$, which is reduced to $[4,10]$.

The reduction of $v_{\mathrm{e} 2}$ and $v_{\mathrm{e} 1}$ has an impact on the evaluation mark $v_{\mathrm{ef}}$ through the numerical constraint $c_{4}$ and reduces it to $\left(v_{\mathrm{e} 1} \times v_{\mathrm{e} 2}\right) \cap D_{v_{\mathrm{ef}}}=([4,10] \otimes[1,2]) \cap$ $[1,30]=[4,20]$.

As an example, let us now consider the case where the user reduces the evaluation mark $v_{\mathrm{ef}}$ to $\leq 6$. This reduction has a direct impact through the numerical constraint $c_{4}$ on the evaluation variable $v_{\mathrm{e} 1}$, which is reduced to $\left(v_{\mathrm{ef}} / v_{\mathrm{e} 2}\right) \cap D_{v_{\mathrm{e} 1}}=([4,6] \oslash[1,2]) \cap[4,10]=[2,6]$, and on 
the evaluation variable $v_{\mathrm{e} 2}$, which is reduced to $\left(v_{\mathrm{ef}} / v_{\mathrm{e} 1}\right) \cap D_{v_{\mathrm{e} 2}}=([4,6] \oslash[2,6]) \cap[1,2]=\left[\frac{2}{3}, 3\right]$.

The reduction of the evaluation variable $v_{\mathrm{e} 1}$ to $[2,6]$ is reflected through the mixed constraint $c_{2}$ on the design variable $v_{\mathrm{d} 1}$, which is reduced to $\|$. This reduction has no impact.

The solution corresponding to $v_{\mathrm{d} 3}<20$ with an evaluation mark $v_{\mathrm{ef}} \leq 6$ is $v_{\mathrm{d} 1}=\|, v_{\mathrm{d} 2}=\perp$.

\subsection{Interests and limits}

The first operating mode matches the expectations of the users: the design of the solution works well and the evaluation mark is computed after each user input in order to reach a final value. This operating mode allows a 'whatif' process in order to observe the impact of different choices on the relevance of the solutions and, therefore, it is very good for comparing different solutions.
The second operating mode can help the user solve her/ his problem by choosing 'by her/himself' coherent values for the negotiable design variables $\left\{V_{\mathrm{d}}\right\}$. However, it can lead to inconsistencies, which derive from $2 \mathrm{~B}$-consistency propagation. Like arc-consistency, 2B-consistency takes the numerical constraints into account sequentially.

Let us consider the previous example, the constraint $c_{2}$ of which is now simplified, as presented in table 2 and illustrated in figure 5 , in order to illustrate this particular

Table 2. Simplified $c_{2}$ constraint.

\begin{tabular}{lcc}
\hline & $c_{2}$ & \\
\hline$v_{\mathrm{d} 1}$ & $v_{\mathrm{d} 2}$ & $v_{\mathrm{e} 1}$ \\
\hline$\perp$ & $\perp$ & {$[5,10]$} \\
$\|$ & $\|$ & {$[1,5]$} \\
\hline
\end{tabular}

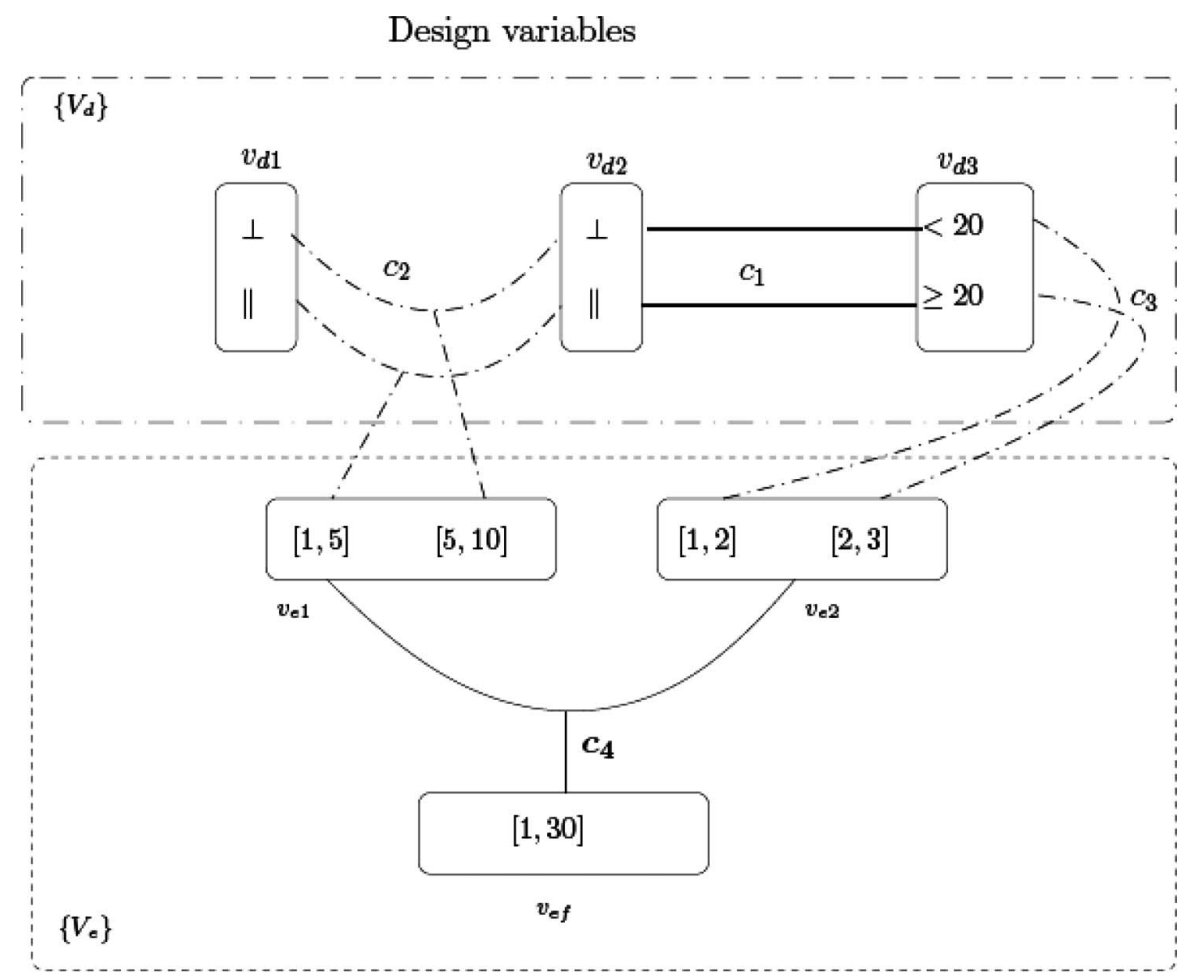

Variables of evaluation

\section{Caption:}

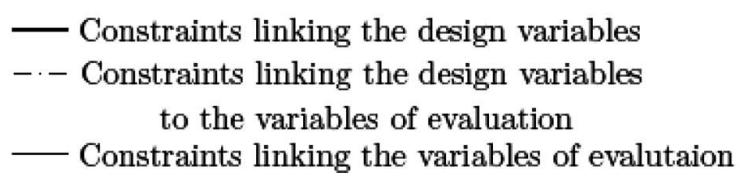

Figure 5. Example of the limit of the second operating mode. 
limit. Now, at the start, as no choice has been made, the evaluation mark $v_{\mathrm{ef}}$ equals $v_{\mathrm{ef}}=v_{\mathrm{e} 1} \times v_{\mathrm{e} 2}=[1,10] \times$ $[1,3]=[1,30]$.

For instance, if the user reduces the evaluation mark $v_{\mathrm{ef}}$ to $<2$, this reduction has a direct impact through the numerical constraint $c_{4}$ on the evaluation variable $v_{\mathrm{e} 1}$, which is reduced to $\left(v_{\mathrm{ef}} / v_{\mathrm{e} 2}\right) \cap D_{v_{\mathrm{e} 1}}=([1,2] \oslash[1,3]) \cap[1,10]=$ $\left[\frac{1}{3}, 2\right]$, and on the evaluation variable $v_{\mathrm{e} 2}$, which is reduced to $\left(v_{\mathrm{ef}} / v_{\mathrm{e} 1}\right) \cap D_{v_{\mathrm{e} 2}}=([1,2] \oslash[1,10]) \cap[1,3]=\left[\frac{1}{3}, \frac{2}{3}\right.$.

The reduction of $v_{\mathrm{e} 1}$ to $\left[\frac{1}{3}, 2\right]$ has an impact on the design variables $v_{\mathrm{d} 1}$ and $v_{\mathrm{d} 2}$ through the mixed constraint $c_{2}$. These two design variables are respectively reduced to $\|$ for $v_{\mathrm{e} 1}$ and $\|$ for $v_{\mathrm{d} 2}$. The reduction of $v_{\mathrm{d} 2}$ to $\left[\frac{1}{3}, \frac{2}{3}\right]$ has an impact on the design variable $v_{\mathrm{d} 3}$ through the mixed constraint $c_{3}$ and reduces it to $<20$. The reduction of $v_{\mathrm{d} 2}$ to $\|$ has an impact on the design variable $v_{\mathrm{d} 3}$ through the mixed constraint $c_{1}$ and reduces it to $\emptyset$. Indeed, the pair of values $\left(v_{\mathrm{d} 2}=\|\right.$, $\left.v_{\mathrm{d} 3}<20\right)$ is not a permissible combination. The problem does not have a solution.

Filtering with stronger consistency, such as 3B-consistency (Lhomme 1993), could avoid this problem, but it would be more time consuming and this is incompatible with an interactive aiding-design tool. This drawback can be avoided, however, by adopting a trial and error design.

\section{Real example}

We have developed such an aiding-design tool as part of a European project (project No. G1RD-CT-2002-00835) called VHT (Virtual Heat Treatment). This tool can, on the one hand, help users design heat treatment operations for steel parts and, on the other, evaluate the designed operations. A software mockup can be found on the Web at http://iena.enstimac.fr:20000/cgi-bin/vht.pl.

In this section we first present the industrial problem that is at the heart of our study, then one of the design models constructed with the experts involved in the project and, more specifically, the evaluation function they defined, and we end with an illustration of the two operating modes. The previous example presented in subsection 3.1 can be seen on the Web under the name IJCIM07.

\subsection{Industrial problem}

In order to improve the mechanical properties of steel, in particular the hardness of the surface, metallurgists treat it with a specific heat treatment operation called quenching. This heat treatment operation consists of three steps. The first step consists of heating a part up slowly in a furnace to a specific temperature called the austenite temperature. The second step consists of maintaining the part at this specific temperature to homogenize the steel micro-structure. The last step consists of the rapid cooling of the part by plunging it into a specific medium such as air, water or oil.
The expected effect of this heat treatment is an improvement in the hardness of the surface of the part but, unfortunately, a negative effect, distortion of the part, usually occurs at the same time.

A means of evaluating distortion is behaviour simulation using Finite Element Method Codes (FEM). They make it possible to obtain a quantitative prediction of the distortion at the design stage. However, the tools are costly, time consuming and very complicated to use, due to the mechanical/structural/thermal behaviour and data requirements. The situation is becoming even more difficult in the current context of smaller production series and the high reactivity of the market. Furthermore, the relevance of the solutions can be the result of some expert's interpretation of many (up to 20) modifications of the dimensions, therefore it is very difficult for a non-expert user to compare different solutions. That is why, more often than not, the design of a quenching heat treatment that minimizes distortion relies on the know-how of experts.

There is a real need for metallurgists to make good decisions without the use of behaviour simulations. Only a few recent studies have used experts' know-how to help them to make better decisions: Varde et al. (2003) used an Expert System to model knowledge and design heat treatment operations with a trial and error procedure, whereas Klein et al. (2005) studied different techniques, such as artificial neural networks or fuzzy logic, to develop a prognosis tool. In this European project, two domain knowledge simulation tools have been developed:

- a CBR tool exploiting implicit domain knowledge, which will not be detailed in this paper. With this tool, each past case is described by 250 relevant parameters. A software mockup can be seen on the Web at http://hefaistos.ivf.se/IMS-VHT/GUI/General.html. This kind of tool is very useful for large companies that always have the same kind of parts to treat, such as the automobile industry. For example, the partner Scania is a good representative of this kind of user. Indeed, a CBR needs many, similar past cases to retrieve those that are nearest to that being considered;

- a tool based on constraints exploiting explicit domain knowledge. A software mockup can be seen on the Web at http://iena.enstimac.fr:20000/cgi-bin/vht.pl. This kind of tool is more useful for heat and surface treatment providers that treat a large variety of parts and need experts' advice for all the different kinds of shapes. For example, the partner Metallographica is a good representative of this kind of user.

In order to develop a tool based on constraints, we have extracted and collected experts' knowledge over the last three years. Inspired by certain design literature models, 
such as that of Oliveria et al. (1986), two intermediate reasoning models based on constraints have been constructed (David et al. 2003) and have led to an advanced model (Aldanondo et al. 2005). In this latter model, many of the constraints resulting from experts' know-how have been validated by other academic partners of the project. These validations have been done either by simulations with FEM codes or by real experiments.

In our application, the experts defined the relevance of the solutions as an evaluation mark $V_{\text {ef }}$ corresponding to a qualitative intensity of distortion $I_{\mathrm{f}}$. As the characterization of a unique intensity of distortion $I_{\mathrm{f}}$ is very complicated to identify for any kind of part, the experts shared out the different shapes to several families of parts, such as axes, disc or gears. First, they focused on the family of axes, which is most often treated by the metallurgists involved in the project.

The definition of a unique intensity of distortion $I_{\mathrm{f}}$ is also very difficult to estimate for a particular part family because the geometry of the part can consist of several features. For instance, an axis can have holes, shoulders and/or variations of thickness, which all have a different impact on the intensity of the distortion $I_{\mathrm{f}}$. Experts have established that the unique intensity of distortion $I_{\mathrm{f}}$ for axes must be decomposed into five distortion intensities, denoted $I_{\mathrm{f}}^{i}$, corresponding to five distortion components gathered in a distortion vector, denoted $D_{i}$ (Lamesle et al. 2005). These five distortion intensities are

- $I_{\mathrm{f}}^{1}$, corresponding to the intensity of distortion following a 'banana' shape. For some reason, due essentially to the direction of the quenching medium and the existence of dissymmetry, an axis can be deformed as a banana, as shown in figure 6;

- $I_{\mathrm{f}}^{2}$, corresponding to the intensity of distortion following a 'spool-barrel' shape. For metallurgical reasons, an axis can be deformed as a spool or a barrel, as shown in figure 7 ;

- $I_{\mathrm{f}}^{3}$, corresponding to the intensity of distortion following a 'spacing-tightening' shape. If there is at least one hole at one of the extremities of the axis, a spacing or a tightening can be observed, as shown in figure 8;
- $I_{\mathrm{f}}^{4}$, corresponding to the intensity of distortion following an 'ovalization' shape. If there is a circular hole along the axis and if the axis is not supported, this circular hole can become oval, as shown in figure 9;

- $I_{\mathrm{f}}^{5}$, corresponding to the intensity of distortion following an 'umbrella' shape. If there is a shoulder on the axis, this shoulder can be deformed as an 'umbrella', as shown in figure 10.

Experts have decided to give a range of $[1,1000]$ to each intensity of distortion $I_{\mathrm{f}}^{i}$ : a value of 1 corresponds to the lowest intensity of distortion, whereas a value of 1000 is the highest intensity.

\subsection{Knowledge model}

The knowledge model is composed of two parts: the first is used to design the heat treatment operation and the second to compute the evaluation function, as shown in figure 11 .

Experts have defined the evaluation function for each intensity of distortion $I_{\mathrm{f}}^{i}$ as the product of the potential intensity of distortion $I_{\mathrm{p}}^{i}$ using a set of evaluation variables $v_{\mathrm{e} j}$ :

$$
I_{\mathrm{f}}^{i}=I_{\mathrm{p}}^{i} \times v_{\mathrm{e} 1} \times \cdots \times v_{\mathrm{e} j}
$$

A potential distortion intensity $I_{\mathrm{p}}^{i}$ is associated with each distortion intensity $I_{\mathrm{f}}^{i}$ and allows a first evaluation of the corresponding distortion intensity $I_{\mathrm{f}}^{i}$. A set of 30 design variables $v_{\mathrm{d}}$, denoted $P_{1}$, are used to compute the value of

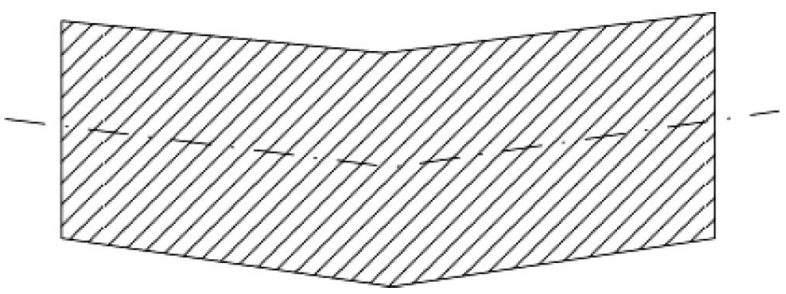

Figure 6. 'Banana' distortion component.

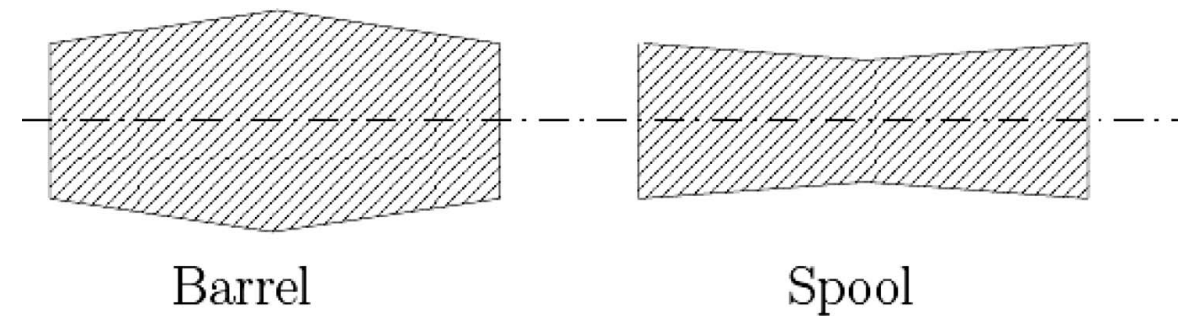

Figure 7. 'Spool-barrel' distortion component. 


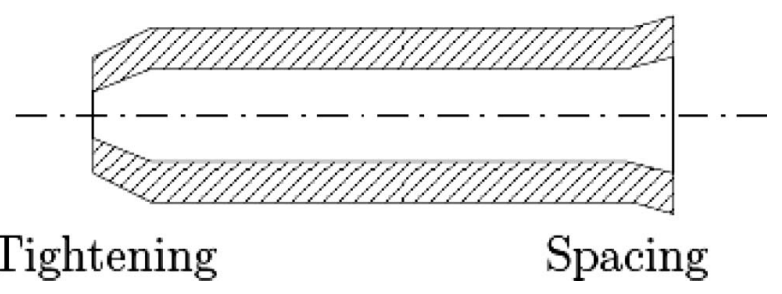

Figure 8. 'Spacing-tightening' distortion component.

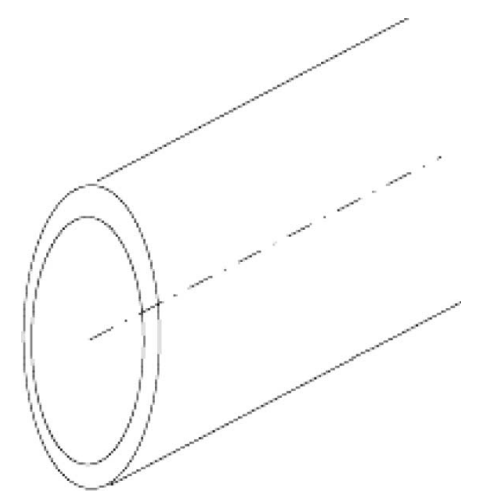

Figure 9. 'Ovalization' distortion component.

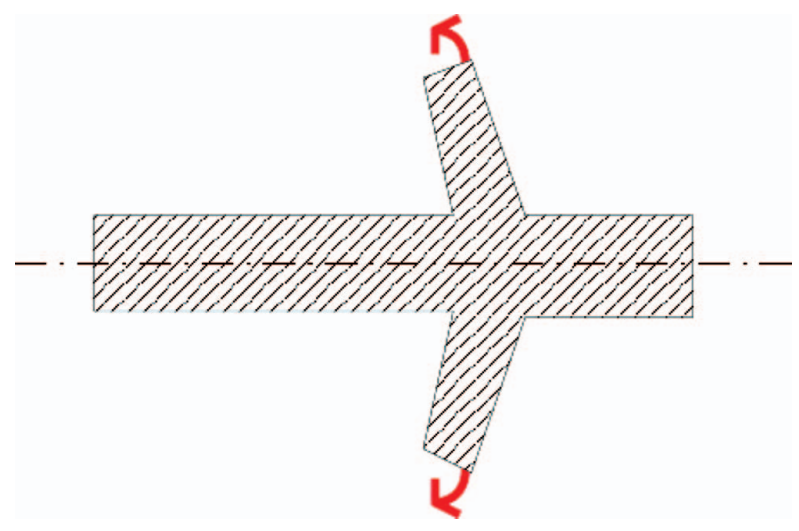

Figure 10. 'Umbrella' distortion component.

the five potential intensities of distortion $I_{\mathrm{p}}^{i}$. These design variables mainly characterize:

- the axis geometry: thickness, diameter, existence of shoulders and holes, etc.;

- the resources used for the operation:

- the position and the wedging of the axis: suspended, supported, etc.,

- the direction of the quenching medium, either perpendicular or parallel to the axis,
- the direction of gravity, either perpendicular or parallel to the axis,

- the steel: $30 \mathrm{CrNiMo} 8,42 \mathrm{CrMo} 4,90 \mathrm{MnV} 8$, etc.

For instance, let us consider the compatibility table 3, which allows us to compute the potential intensity of distortion following a 'banana' shape $I_{\mathrm{p}}^{1}$. This intensity is characterized by the direction of the quenching medium $D_{\mathrm{qm}}$, by the direction of gravity $D_{\mathrm{g}}$ and by the position and wedging of the axis $P_{\mathrm{a}}$. Each value of this potential intensity corresponds to a combination of values of the subset of the designed variables belonging to $P_{1}$.

It can be seen from figure 11 that each potential distortion intensity $I_{\mathrm{p}}^{i}$ results from a different part of the design model.

Around 30 evaluation variables $v_{\mathrm{e} j}$ increase or decrease the value of the potential distortion intensity $I_{\mathrm{p}}^{i}$ in order to compute the evaluation mark $I_{\mathrm{f}}^{i}$. A set of 70 design variables $v_{\mathrm{d}}$, denoted $P_{2}$, are used to compute the value of these evaluation variables $v_{\mathrm{e} j}$. Experts have apportioned these variables into six groups, characterizing:

- the quenching medium: gas, water, air, oil, drasticity, temperature, etc.;

- load preparation: basket permeability, symmetry around the part, etc.;

- the general geometry of the axis: thickness variation, degree of dissymmetry, etc.;

- the metallurgical characteristics: material trempability, carburizing, etc.;

- the material history: forming process, relaxation, etc.;

- the heating cycle: furnace class, heating speed, etc.

For instance, let us consider the compatibility table 4, which allows us to compute the evaluation variable $v_{\mathrm{e} 12}$. This evaluation variable is characterized by the quenching medium $Q m$ and by the thickness variation $T v$ of the part. Each value of the evaluation variable corresponds to a combination of values of the subset of designed variables belonging to $P_{2}$, showing that a liquid quenching fluid is better than a gas fluid and that a small thickness variation has a small impact on the intensity of distortion.

It is worth stressing that a very small subset of design variables belongs simultaneously to subsets $P_{1}$ and $P_{2}$ and that the constraints between the variables of $P_{1}$ and $P_{2}$ correspond to technical feasibilities. $P_{1}$ and $P_{2}$ are not differentiated for users: they reduce indifferently the domains of the design variables belonging to $P_{1}$ and $P_{2}$ indifferently.

As each distortion intensity $I_{\mathrm{f}}^{i}$ must belong to the interval $[1,1000]$ and is computed from the product of its potential distortion attribute $I_{\mathrm{p}}^{i}$ by a specific set of evaluation variables $v_{\mathrm{e} j}$, the influence of the two subsets of design 


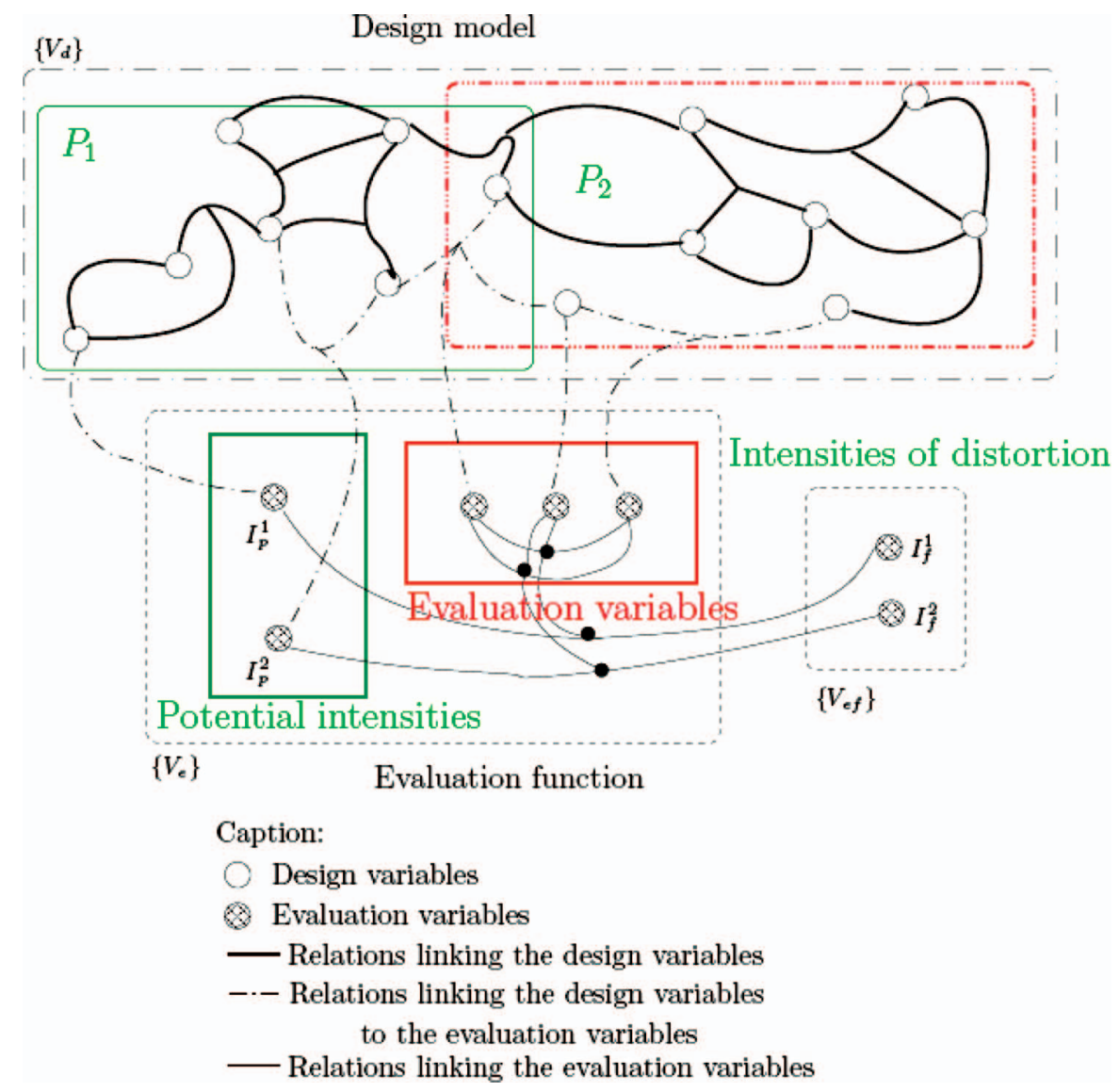

Figure 11. Architecture of the knowledge model.

Table 3. Characterization of the potential intensity of the 'banana'-shape distortion.

\begin{tabular}{lllc}
\hline$D_{\mathrm{qm}}$ & $D_{\mathrm{g}}$ & $P_{\mathrm{a}}$ & $I_{\mathrm{p}}^{\mathrm{l}}$ \\
\hline Parallel & Parallel & Suspended & 1 \\
Parallel & Parallel & Supported & {$[1,2.3]$} \\
Parallel & Perpendicular & Good chock & {$[2.3,4.3]$} \\
Parallel & Perpendicular & Bad chock & {$[4.3,6.2]$} \\
Perpendicular & Parallel & Suspended & {$[7.9,8.9]$} \\
Perpendicular & Parallel & Supported & {$[8.9,10.2]$} \\
Perpendicular & Perpendicular & Good chock & {$[10.2,11.5]$} \\
Perpendicular & Perpendicular & Bad chock & {$[12.8,14.1]$} \\
\hline
\end{tabular}

variables $P_{1}$ and $P_{2}$ have been analysed by experts. Their influences result from experts' know-how:

- the influence of $P_{1}$ on the final intensity of distortion has been fixed at 30\%: each potential distortion attribute $I_{\mathrm{p}}^{i}$ belongs to the interval [1,20];

- the influence of $P_{2}$ has been fixed at $70 \%$ : the product of all the evaluation variables $v_{\mathrm{e} j}$ belongs to the interval
Table 4. Characterization of the evaluation variable $v_{\mathrm{e} 12}$.

\begin{tabular}{llc}
\hline$T v$ & $Q m$ & $v_{\mathrm{e} 12}$ \\
\hline$>1.5$ & Water & {$[10,12]$} \\
{$[1,1.5]$} & Water & {$[8,10]$} \\
$<1$ & Water & {$[7,8]$} \\
$>1.5$ & Oil & {$[5,7]$} \\
{$[1,1.5]$} & Oil & {$[3,5]$} \\
$<1$ & Oil & {$[2.5,3]$} \\
$>1.5$ & Air & {$[1.8,2.5]$} \\
{$[1,1.5]$} & Air & {$[1.2,1.8]$} \\
$<1$ & Air & 1.2 \\
\hline
\end{tabular}

$[1,50]$. As subset $P_{2}$ is composed of six groups, the influence of each was also determined by the experts:

- $35 \%$ for the group characterizing the quenching medium,

- $20 \%$ for the group characterizing the load preparation,

- $15 \%$ for the group characterizing the general geometry, 
- $\quad 15 \%$ for the group characterizing the metallurgical characteristics,

- $10 \%$ for the group characterizing the material history,

- and 5\% for the group characterizing the heating cycle.

To synthesize, the final reasoning model is composed of

- five distortion components $D_{i}$;

- five potential distortion intensities $I_{\mathrm{p}}^{i}$ belonging to the interval $[1,20]$;

- five distortion intensities $I_{\mathrm{f}}^{i}$ equal to the product of $I_{\mathrm{p}}^{i}$ and $v_{\mathrm{e} j}$ and belonging to the interval $[1,1000]$;

- around 90 design variables with 30 belonging to $P_{1}$, which allow us to compute the potential distortion intensity $I_{\mathrm{p}}^{i}$, and 70 belonging to $P_{2}$, which are linked to a set of 30 evaluation variables $v_{\mathrm{e} j}$ to compute the evaluation mark of the designed solution;

- around 10 discrete, 30 mixed and 10 continuous compatibility tables;

- around 60 mathematical constraints to compute the final evaluation marks and to allow the use of the model with the two operating modes.

\subsection{Operating modes}

The first operating mode lives up to the metallurgists' expectations: the design choices are well reflected in the variable domains, laid to a consistent heat treatment operation, and the intensities of distortion $I_{\mathrm{f}}^{i}$ of each distortion component are in agreement with the prediction of the metallurgists.

After each user's input, the distortion intensities $I_{\mathrm{f}}^{i}$ are progressively reduced. We can see from figure 12 that the evolution of the distortion intensity follows one of the distortion components: the 'spool-barrel'. Before any choice is made, this intensity belongs to $[1,1000]$ (time (0)) and decreases after each user input:

(i) description of the part geometry (time (1));

(ii) description of the load preparation (time (2));

(iii) description of the material history (time (3));

(iv) description of the metallurgical characteristics (time (4));

(v) and description of the quenching fluid (time (5)).

Therefore, the simultaneous design/evaluation permits a 'what-if' decision aid.

If, in the previous example, a threshold is given to the intensity of distortion of the 'spool-barrel' component after a complete description of the material history (time 3), e.g. distortion $<50$ as shown in figure 13 , the selection of a

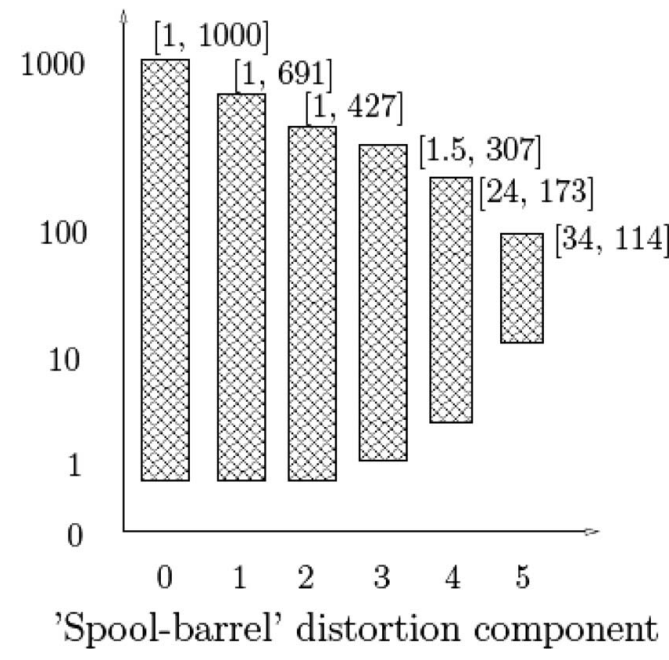

Figure 12. Evolution of a distortion intensity.

quenching fluid is done automatically by the system: oil. As pointed out in section 3.3, the second operating mode can lead to inconsistent solutions if the thresholds on the distortion intensities $I_{\mathrm{f}}^{i}$ are too strong.

A drawback of the model has also been identified by the experts: in all the models developed using experts, only the minimization of the distortion is actually taken into account. Then, when a user designs a heat treatment operation in order to minimize the intensity of distortion, the solution reached effectively minimizes the distortion, but sometimes does not improve the mechanical properties of the part. We have to improve our models by adding another relevant component representing the improvement of the mechanical properties. The user will then have to come to an agreement concerning the improvement of the mechanical properties and the minimization of the resulting distortion.

\subsection{Synthesis}

In order to develop the reasoning models presented in the previous section, we have extracted and collected experts' knowledge over three years. During this time, an advanced model has been constructed for a specific family of parts, axes, and it still needs some improvements before being completed.

The extraction and validation of the knowledge are always lengthy and difficult and even more so when the knowledge is not always available, accessible, easy to validate and to formalize. Furthermore, the problem of maintenance of the models is crucial: some knowledge is stable and some is not. In order to maintain the models we have to identify both the stability of the knowledge and the necessary frequency of maintenance. 


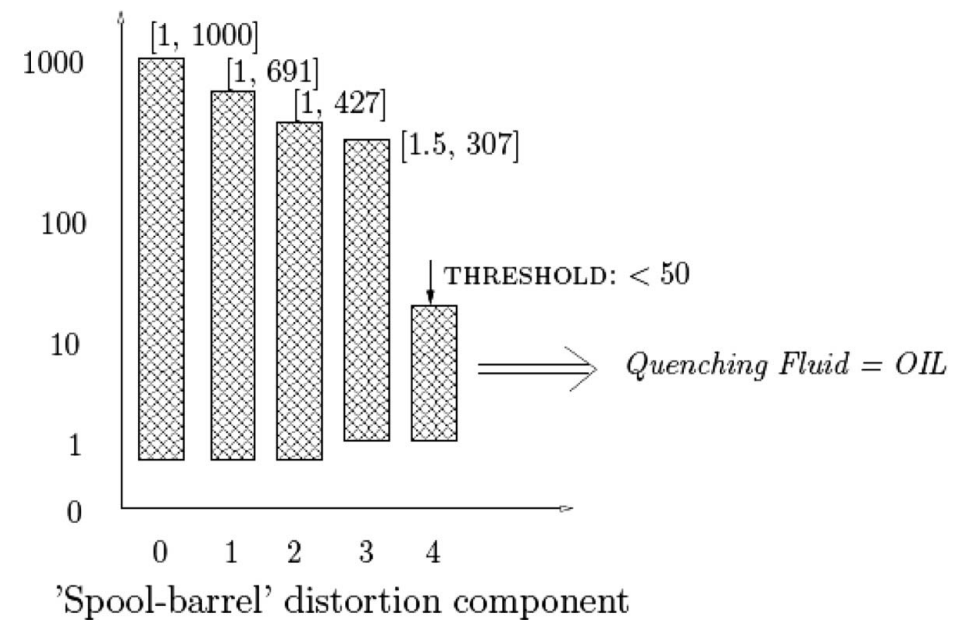

Figure 13. Selection of a negotiable variable.

This kind of tool is easier to develop if the object of study is fairly routine because the knowledge is more stable and therefore easier to identify, to extract and also to maintain. This was the case in our application.

\section{Conclusion}

The aim of this paper has been to show how the relevance of solutions can be estimated or evaluated in an interactive aiding-design process in order to help users to make better decisions.

First we compared how the solutions can be evaluated by two means: behaviour simulations and domain knowledge simulations. Behaviour simulations retrieve the 'best' solution following several criteria. It is the user who has to determine the relevance of the solution. For domain knowledge simulations, CBR, ES and CSP, there is usually no method for evaluating the solutions for a non-expert user. We have therefore concentrated our study on domain knowledge simulations in order to fill this gap.

We identified three different methods of evaluating solutions in domain knowledge simulations:

- identification of a small set of relevant and independent data. In this case, the evaluation is embodied in the system;

- definition of an explicit evaluation function defined by experts which must be added to the system;

- learning from past experiments using an ANN which is added to the system;

- or regression analysis.

We have focused on constraints-based systems because of their consistent properties, their ability to model experts' knowledge and for their ability to be easily understood. As this kind of model is based on the extraction of experts' know-how, for its translation into constraints and its validation, we have assumed that experts are able to determine the set of data to be taken into account and how to combine them in order to define an explicit evaluation function. The constraints-based system is then composed of two different parts: one which helps the user to design her/ his solution, and the other which evaluates the solution.

Being based on constraints, which do not have propagating directions, the knowledge model can be used in two operating modes. The first, evaluation of a solution, consists of interactively inputting restrictions on the design variables in order to find a coherent solution and of simultaneously evaluating the solution. The second mode, choices deduced from an evaluation, consists of inputting restrictions on non-negotiable design variables and a threshold on the evaluation in order to achieve values for the negotiable design variables.

This kind of approach has been used in a real problem to help users design and evaluate heat treatment operations. This tool is appreciated by the users because:

- it is very useful for comparing solutions, although it is somewhat weak for comparing different solutions in an absolute way;

- it is very useful for observing the consequences of a decision in a "what-if' process;

- although the second operating mode is somewhat tricky to use, it is much appreciated by users in helping them complete a heat treatment operation design.

We are currently working on improvements to our design models in order to take into account the mechanical 
properties of steel and on improvements of the ergonomics of the Web mockup in order to make it more user-friendly.

\section{Acknowledgements}

This work was partly funded by the European Commission through the IMS project. The authors wish to acknowledge the Commission for their support. We also wish to acknowledge our gratitude and appreciation to all the VHT project partners (especially EMTT (France), SCC (France), Metallographica (Spain), Scania (Sweden) and IWT (Germany)) for their contribution during the development of the various ideas and concepts presented in this paper. This paper is an extended version of that in the proceedings volume from the 12th IFAC International Symposium on Information Control Problems in Manufacturing (INCOM'06) (ISBN: 978-0-08-044654-7, Elsevier Science, Saint Etienne, France, December 2006).

\section{References}

Aldanondo, M., Vareilles, E., Lamesle, P., Hadj-Hamou, K. and Gaborit, P., Interactive configuration and evaluation of a heat treatment operation, in Workshop on Configuration, International Joint Conference on Artificial Intelligence, 2005.

Banks, J., Carson, J. and Nelson, B., Discrete-event System Simulation (International Series in Industrial and Systems Engineering), 1984 (Prentice-Hall: Englewood Cliffs, NJ).

Bouzeghoub, M., Gardarin, G. and Metais, E., Database design tools: an expert system approach, in 11th International Conference on Very Large Data Bases, 1985, pp. 82-95.

Buchanan, B-G. and Shortliffe, E-H., Rule-based Expert Systems, the Mycin Experiments of a Stanford Heuristic Programming Project, 1984 (Addison-Wesley: Reading, MA).

David, P., Veaux, M., Vareilles, E. and Maury, J., Virtual heat treatment tool for monitoring and optimising heat treatment, in 2nd International Conference on Thermal Process Modelling and Computer Simulation, 2003.

Dechter, R., From local to global consistence. Artif. Intell., 1992, 87-107. Faltings, B., Arc consistency for continuous variables. Artif. Intell., 1994, $363-376$.

$\mathrm{Fu}, \mathrm{M}$., Optimization for simulation: theory vs. practice. INFORMS J. Comput., 2002, 14, 192-215.
Gelle, E., On the generation of locally consistent solution spaces in mixed dynamic constraint problems. PhD thesis, École Polytechnique Fédérale de Lausanne, Suisse, 1998.

Huang, G.Q., Design for X-Concurrent Engineering Imperatives, 1996 (Springer: Berlin)

Klein, D., Thoben, K-D. and Nowag, L., Using indicators to describe distortion along a process chain, in International Conference on Distortion Engineering, 2005.

Kolodner, J., Case-based Reasoning, 1993 (Morgan Kaufmann).

Lamesle, P., Vareilles, E. and Aldanondo, P., Towards a KBS for a qualitative distortion's prediction for heat treatments, in International Conference on Distortion Engineering, 2005.

Lhomme, O., Consistency techniques for numeric CSP, in International Joint Conference on Artificial Intelligence, 1993, pp. 232-238.

Mackworth, A.K., Consistency in networks of relations. Artif. Intell., 1977, 99-118.

Magrab, E-B., Integrated Product and Process Design and Development, 1997 (CRC Press: New York).

Maher, M., Zhang, D. and Balachandran, M., Case-based Reasoning in Design, 1995 (Lawrence Erlbaum Associates).

Minsky, M. and Papert, S., Perceptrons, 1969 (MIT Press: Cambridge).

Montanari, U., Networks of constraints: fundamental properties and application to picture processing. Inf. Sci., 1974, 7, 95-132.

Moore, R.E., Interval Analysis, 1966 (Prentice-Hall: Englewood Cliffs, NJ).

Oliveira, M., Moreira, A., Loureiro, A.P., Denis, S. and Simon, A., Effet du mode de refroidissement sur les déformations produites par la trempe martensitique, in 5th Congrès International sur les Traitements Thermiques des Matériaux, 1986.

Riesbeck, E-1. and Shank, R., Inside Case-based Reasoning, 1989 (Lawrence Erlbaum Associates: NJ).

Szabo, B. and Babuska, I., Finite Element Analysis, 1991 (Wiley: New York).

Tsang, E., Foundations of Constraints Satisfaction, 1993 (Academic Press: London).

Tsatsoulis, C., A review of artificial intelligence in simulation. $A C M$ SIGART Bull., 1990, 2, 115-121.

Varde, A., Maniruzzaman, M., Rundensteiner, E. and Richard, D., The QuenchMiner expert system for quenching and distortion control, in ASM's HTS-03, 2003, pp. 174-183.

Vareilles, É., Conception et approches par propagation de contraintes: contribution la mise en oeuvre d'un outil d'aide interactif. $\mathrm{PhD}$ thesis, École des mines, Albi-Carmaux, France, 2005.

Vernat, Y., Formalisation et qualification de modèles par contraintes en conception préliminaire. PhD thesis, École Nationale des Arts et Métier, Bordeaux, France, 2004 